\title{
Reflets
}

Revue d'intervention sociale et communautaire

\section{Penser le pluralisme des francophonies minoritaires canadiennes : de la logique identitaire à la question sociale}

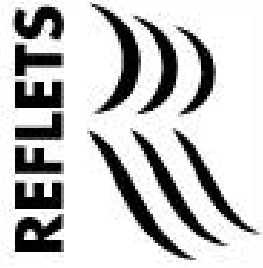

\section{Stéphanie Garneau}

Volume 16, numéro 2, 2010

L'intervention sociale en contextes minoritaires : penser la complexité et la multiplicité des processus de minorisation

URI : https://id.erudit.org/iderudit/1000313ar

DOI : https://doi.org/10.7202/1000313ar

Aller au sommaire du numéro

Éditeur(s)

Reflets, Revue d'intervention sociale et communautaire

ISSN

1203-4576 (imprimé)

1712-8498 (numérique)

Découvrir la revue

Citer cet article

Garneau, S. (2010). Penser le pluralisme des francophonies minoritaires canadiennes : de la logique identitaire à la question sociale. Reflets, 16(2), 22-56. https://doi.org/10.7202/1000313ar

\section{Résumé de l'article}

Malgré les acquis obtenus par les Francophones vivant en situation minoritaire au Canada, les inégalités sociales entre les minorités francophones et les majorités anglophones persistent. Parallèlement, d'autres catégories et groupes sociaux (femmes, communautés ethniques, etc.) se trouvent au sein des francophonies minoritaires et revendiquent des droits, en conformité avec la Loi sur le multiculturalisme, sur la base de leur particularité identitaire. Que faire lorsque les intérêts des uns entrent en conflit avec les intérêts des autres? Cet essai propose un cadre de réflexion susceptible d'orienter nos recherches sur " les minorités au sein des minorités francophones ". Il se penche d'abord sur les raisons pour lesquelles les travaux portant sur la complexité des identités/inégalités sociales au sein des minorités francophones canadiennes ont émergé tardivement. Il tente ensuite, à partir de l'approche de l'intersectionnalité, d'analyser les effets du multiculturalisme sur les francophonies en situation minoritaire. Est-ce que le multiculturalisme canadien nuit aux intérêts des minorités francophones? L’idée défendue est qu'il n'y a pas lieu d'opposer les francophonies minoritaires aux autres minorités, mais qu'il est plus fécond de penser la simultanéité des processus pluriels de domination en déplaçant la polémique identitaire sur le terrain des inégalités sociales. Il est suggéré que c'est l'ambiguïté même du multiculturalisme canadien qui permet, paradoxalement, ce renversement de perspective.
Tous droits réservés @ Reflets, Revue d’intervention sociale et communautaire, 2010
Ce document est protégé par la loi sur le droit d'auteur. L'utilisation des services d'Érudit (y compris la reproduction) est assujettie à sa politique d'utilisation que vous pouvez consulter en ligne. 


\section{Penser le pluralisme des francophonies minoritaires canadiennes : de la logique identitaire à la question sociale ${ }^{1}$}

Stéphanie Garneau

Professeure, École de service social, Université d'Ottawa

\section{Résumé}

Malgré les acquis obtenus par les Francophones vivant en situation minoritaire au Canada, les inégalités sociales entre les minorités francophones et les majorités anglophones persistent. Parallèlement, d'autres catégories et groupes sociaux (femmes, communautés ethniques, etc.) se trouvent au sein des francophonies minoritaires et revendiquent des droits, en conformité avec la Loi sur le multiculturalisme, sur la base de leur particularité identitaire. Que faire lorsque les intérêts des uns entrent en conflit avec les intérêts des autres? Cet essai propose un cadre de réflexion susceptible d'orienter nos recherches sur «les minorités au sein des minorités francophones». Il se penche d'abord sur les raisons pour lesquelles les travaux portant sur la complexité des identités/inégalités sociales au sein des minorités francophones canadiennes ont émergé tardivement. Il tente ensuite, à partir de l'approche de l'intersectionnalité, d'analyser les effets du multiculturalisme sur les francophonies en situation minoritaire. Est-ce que le multiculturalisme canadien nuit aux intérêts des minorités francophones? L'idée défendue est qu'il n'y a pas lieu d'opposer les francophonies minoritaires aux autres 
minorités, mais qu'il est plus fécond de penser la simultanéité des processus pluriels de domination en déplaçant la polémique identitaire sur le terrain des inégalités sociales. Il est suggéré que c'est l'ambiguiité même du multiculturalisme canadien qui permet, paradoxalement, ce renversement de perspective.

Mots clés : minorités francophones, multiculturalisme, intersectionnalité, inégalités sociales, reconnaissance identitaire

\section{Abstract}

In spite of social gains obtained by French-speaking people living as a minority in Canada, social inequalities between minority Francophones and majority Anglophones persist. However, other social categories and groups (women, ethnic communities, etc) within minority Francophones, in conformity with the Canadian Multiculturalism Act, assert some rights on the basis of their particular identity. What action is required when the interests of one group enter in conflict with the interests of the others? This essay proposes a framework of thinking likely to direct our research on "minorities within minority Francophones". First, it focuses on the reasons for which research relating to the complexity of identities/social inequalities within minority Francophones in Canada was late in emerging. Then, it tries, starting from intersectional analysis, to analyze the effects of multiculturalism on minority Francophones. Does Canadian multiculturalism harm the interests of minority Francophones? The idea defended in this essay is not to put minority Francophones in conflict with other minority groups, but to think the simultaneity and the plurality of domination's processes by moving from the identity polemic to the ground of social inequalities. It is suggested that it is the ambiguity of the Canadian Multiculturalism Act that allows, paradoxically, this reversal of perspective.

Key words: minority Francophones, multiculturalism, intersectional analysis, social inequalities, identity recognition 
Le concept de minorité est avant tout sociologique et politique. En effet, le statut de minoritaire ne se réduit pas à une simple différence, ni à une question statistique ou démographique. Il repose plutôt sur une relation sociale qui met en scène un dominant et un dominé :

Tous ont en commun leur forme de rapport à la majorité, l'oppression. Oppression économique d'abord, oppression légale (ou coutumière) ensuite. Les formes de ce rapport d'oppression économique et sociale sont différentes selon les groupes mais constantes : ces groupes se définissent par leur état de dépendance au groupe majoritaire. Ils sont, au sens propre du terme, en état de minorité. Minorité :être moins (Guillaumin, 1972, p. 86)

Ainsi, sur la base d'une supposée différence - physique ou culturelle, souvent les deux -, les personnes qui composent un groupe minoritaire sont traitées différemment et de manière inégale par rapport au reste de la population. En conséquence, elles considèrent qu'elles font face à des actes de discrimination relatifs à leur identité collective dans différentes sphères de leur vie quotidienne.

Depuis l'adoption de la Charte canadienne des droits et libertés en 1982, les minorités francophones canadiennes ont acquis plusieurs droits en matière linguistique. Néanmoins, les inégalités entre minorités francophones et majorités anglophones n'ont pas disparu. Par exemple, les Francophones vivant en situation minoritaire et souffrant de troubles de santé mentale rencontrent encore de sérieux obstacles à l'obtention de services en français, dans un champ de la santé où le travail de guérison emprunte souvent la voie de la thérapie, et donc du langage (Boudreau, 1999). Toujours sur le plan de la santé, des études réalisées auprès de certaines minorités au Canada montrent que la proportion des personnes obtenant un faible niveau sur l'indice de l'état de santé est plus élevée chez les Francophones en situation minoritaire que chez les groupes immigrants de langue anglaise 
(17,8 \% contre 12,7 \%) (Bouchard et collab., 2006, p. 519). De plus, les Francophones de l'Ontario présenteraient un taux d'analphabétisme presque deux fois plus élevé (25\%) que celui des Ontariens anglophones (Denault et Cardinal, 1999, p. 94; Coderre et Dubois, 2000, p. 64), et leur taux de chômage serait également plus élevé (Denault et Cardinal, 1999, p. 94). Encore aujourd'hui, les jeunes Francophones de la province de l'Ontario feraient partie des catégories d'étudiants sous-représentés dans l'enseignement supérieur, aux côtés notamment des Autochtones et des jeunes avec un handicap (Rae, 2005, p. 6). Aux côtés de la question sociale touchant les francophonies minoritaires se trouve aussi la question identitaire, laquelle mobilise autant, sinon plus, d'énergie revendicatrice de la part des communautés concernées. En effet, avec plus ou moins d'empressement selon les milieux, car c'est la survie même de la langue française, d'une culture, d'une mémoire et d'une identité collective qui se trouve menacée, une partie des communautés se mobilise ${ }^{2}$.

Cela étant posé, les aspirations à la justice sociale et à la reconnaissance de leur identité au sein de la société canadienne ne sont pas le seul fait des francophonies en situation minoritaire. D'autres minorités — définies sur la base de l'ethnicité, du genre, de l'orientation sexuelle, de l'âge, du handicap — présentent aussi, en conformité avec la Loi sur le multiculturalisme, des demandes sociales. Or, une revue de la littérature nous permet de constater que les recherches visant à penser les minorités au sein des francophonies minoritaires canadiennes sont récentes et encore peu nombreuses ${ }^{3}$. Plusieurs travaux continuent d'appréhender les divers groupes minoritaires de façon distincte, voire dans une logique concurrentielle. Mais que faire dans cette dernière perspective si les intérêts des uns entrent en conflit avec ceux des autres? Doit-on privilégier les intérêts du groupe majoritaire, soi-disant représentant d'un supposé universalisme?

Cet essai porte sur les réflexions appelées par cette réalité sociale. Dans un premier temps, nous analyserons les raisons pour lesquelles les travaux traitant de la complexité des identités/ inégalités sociales au sein des minorités francophones canadiennes ont émergé tardivement. Si l'accroissement relativement nouveau 
"...en faisant des minorités francophones canadiennes des minorités comme les autres, la politique sur le multiculturalisme ne nuit-elle pas aux intérêts des francophonies minoritaires?" des flux d'immigration dans les communautés francophones peut sans contredit en être l'une des explications, du moins en ce qui concerne la dimension ethnique de la différenciation sociale, elle ne nous parait pas exclusivement déterminante pour expliquer ce constat. Deux autres raisons, en effet, seront explorées ici. La première concerne l'adoption de la Loi sur les langues officielles et la Politique sur le multiculturalisme, lesquelles ont concouru à l'ethnicisation de l'identité canadienne-française, dans une logique concurrentielle avec d'autres groupes minoritaires qui ne favorise pas la prise en compte simultanée des identités. La seconde concerne le champ scientifique et renvoie au fait que c'est par le biais des études féministes de l'intersectionnalité, dont la reconnaissance fut tardive dans le milieu académique, que l'analyse des processus multiples de minorisation a fait son entrée dans les sciences sociales pratiquées en francophonies minoritaires.

Ce détour par les effets de la politique sur le multiculturalisme sur la définition et l'appréhension scientifiques de l'identité canadienne-française nous permettra de souligner la question qu'elle contient en creux : en faisant des minorités francophones canadiennes des minorités comme les autres, la politique sur le multiculturalisme ne nuit-elle pas aux intérêts des francophonies minoritaires? Dans un deuxième temps, nous interrogerons donc les termes de cette question, cela en recourant aux théories féministes de l'intersectionnalité. Nous défendrons l'idée qu'il n'y a pas lieu d'opposer les francophonies minoritaires aux minorités ethniques - pas plus d'ailleurs qu'aux minorités de genre, d'orientation sexuelle, ou autres - , mais qu'il apparaît plus fécond de penser la simultanéité des processus pluriels de minorisation en déplaçant la polémique identitaire sur le terrain des inégalités sociales. Nous verrons que cette alternative est rendue possible par l'ambiguïté même du multiculturalisme canadien : si ce dernier porte en lui les risques d'une ethnicisation des rapports sociaux, il permet en revanche, surtout depuis l'adoption de la Loi sur le multiculturalisme en 1988, une reconnaissance des identités dans le but de combattre les inégalités sociales. Nous espérons ainsi proposer un cadre de réflexion susceptible d'orienter nos recherches sur les minorités au sein des minorités francophones. 


\section{La lente émergence des travaux portant sur la complexité des inégalités/identités en contexte francophone minoritaire}

Au sein des francophonies canadiennes hors Québec, la tardive adoption du vocable «minoritaire» pour se définir trouve une première explication dans les rapports sociohistoriques qui ont participé à la naissance des minorités linguistiques au Canada, ainsi que dans l'adoption de la Loi sur les langues officielles et de la Politique sur le multiculturalisme. Ensemble, ces réalités ont posé les cadres politico-juridiques à l'intérieur desquels se sont définis et se définissent toujours, bien que différemment, les revendications et le discours identitaire dominant des francophonies minoritaires.

\section{Les cadres politico-juridiques structurant le} discours identitaire des francophonies minoritaires : la Loi sur les langues officielles et la Politique sur le multiculturalisme

Faut-il rappeler que les nations sont des «communautés politiques imaginées" (Anderson, 1996) auxquelles participe le processus historique de formation des États? ${ }^{4}$ Aussi, la production de la nation met au jour des pratiques et des politiques en conflits, chacune légitimée par le recours discursif à un ou à des mythes fondateurs d'une histoire et de valeurs communes. Les fondements de la "nation» canadienne n'y font pas exception.

Jusqu'en 1971, année de l'adoption de la politique sur le multiculturalisme, le discours officiel de la nation canadienne renvoyait à deux peuples fondateurs, à savoir les pays colonisateurs que furent la Grande-Bretagne et la France. Ce fondement de la Confédération canadienne sur deux nations, l'une canadiennefrançaise et l'autre canadienne-anglaise, a évidemment impliqué 
un travail d'homogénéisation de ces deux groupes qui a abouti à des mesures diverses : discours omettant la présence antérieure des Autochtones sur le territoire canadien, y compris les rapports de collaboration de toutes sortes pourtant jadis noués avec eux; adoption de politiques visant une immigration blanche et «de type» britannique chez les Canadiens anglais; développement d'une conception culturaliste et primordialiste de la nation chez les Canadiens français qui déplacera dans un premier temps les immigrants du côté canadien-anglais; abolition des écoles françaises dans les provinces à majorité anglophone - où d'ailleurs se vérifiait l'assimilation des immigrants au modèle britannique _ , d'abord au Manitoba en 1896 puis en Ontario en 1913 (Juteau, 1993).

En dépit de cette dualité pour le moins conflictuelle entre Canadiens anglais et Canadiens français, la communauté francophone canadienne jouissait alors d'institutions et d'un certain niveau organisationnel fondé principalement sur l'Église catholique - dont les ramifications transcendaient les frontières provinciales (Thériault, 1994) ${ }^{5}$. Le Canada français existait sans référence aux territoires provinciaux; il était conçu comme le cofondateur du pacte des deux nations à l'origine de la grande nation canadienne. Selon Thériault (1994, p. 22), les francophonies minoritaires étaient - et sont encore - des regroupements nationalitaires. Ni nation, ni groupe ethnique issu de l'immigration, elles sont "des communautés de destin qui ont un niveau d'historicité plus fort que l'ethnie, mais plus faible que la nation».Par niveau d'historicité,Thériault entend le « déploiement au sein d'une collectivité d'une conscience et d'une capacité de faire son histoire» (1994, p. 20). Les regroupements nationalitaires possèdent la plupart des caractéristiques des nations (langue, références culturelles communes), mais n'ont pas de frontières étatiques. Pour autant, elles veulent «faire société".

Avec la modernisation - et la laïcisation — de l'État au cours des années 1960, cette «intention vitale» du Canada français, c'est-à-dire ce "principe instituant autour duquel un groupement humain - en l'occurrence ici le Canada français - constitue une pluralité d'hommes et de femmes en fait social global», s'est 
"A travers le prisme de l'État provincial qui devient alors le cadre privilégié d'action dans plusieurs domaines, les communautés francophones hors Québec perdent leur statut de communauté politique — l'une des nations fondatrices du Canada - pour se transformer en minorités..." évanouie (Thériault et Meunier, 2008, p. 224). À travers le prisme de l'État provincial qui devient alors le cadre privilégié d'action dans plusieurs domaines, les communautés francophones hors Québec perdent leur statut de communauté politique — l'une des nations fondatrices du Canada - pour se transformer en minorités (Juteau-Lee et Lapointe, 1983; Thériault, 1994). De plus, le mouvement nationaliste québécois qui souffle à la même époque participe à la redéfinition de l'idée de la francophonie en Amérique du Nord en la territorialisant à l'intérieur de ses frontières, cela en éjectant du même coup de son projet les Francophones qui se trouvent à l'extérieur. Le mouvement nationaliste québécois fait éclater la mémoire canadienne-française et l'idée de nation cofondatrice du Canada.

L'époque en est d'ailleurs une de crise pour l'unité canadienne. En 1963, le gouvernement fédéral mettra en place la Commission royale d'enquête sur le bilinguisme et le biculturalisme en vue de trouver une nouvelle entente. Les propositions de cette Commission iront dans le sens du maintien de la dualité culturelle du Canada, tout en redonnant une existence aux Autochtones et en accordant de l'attention aux groupes dits ethniques - soient ceux, grandissants, issus de l'immigration. Cependant, le premier ministre canadien Pierre Elliot Trudeau, fervent opposant au nationalisme tant canadien-français que québécois, refusera les recommandations de la Commission pour substituer à l'idée de biculturalisme celle de multiculturalisme. Aussi, avec la Loi sur les langues officielles adoptée en 1969, l'idée du bilinguisme sera maintenue. En revanche, la politique sur le multiculturalisme adoptée en 1971 ne donnera préséance à aucune culture sur les autres. Le discours officiel fera désormais la promotion de l'idéologie pluraliste : le fondement caractéristique et distinctif de l'identité canadienne devient celui de la diversité culturelle dans un contexte bilingue. La loi et la politique seront ensuite toutes deux enchâssées dans la Constitution via la Charte canadienne des droits et libertés en 1982.

Or,

[1] a promulgation de la diversité culturelle comme objectif désirable passait sous silence les colonialismes français et britannique, occultait 
"Avec l'adoption de la politique sur le multiculturalisme, les minorités francophones deviennent des minorités comme les autres en compétition pour les ressources de l'État.» l'existence des Premières Nations, niait le statut national des Francophones, séparait langue et culture, taisait les inégalités politiques et économiques entre les diverses communautés ethniconationales, occultait la discrimination subie par les femmes et autres groupes racisés [...] et culturalisait la définition des enjeux. Bref, nous sommes en face d'une représentation de la nation canadienne qui masque les rapports de pouvoir sur lesquels elle s'est érigée. (Juteau, 1993, p. 63)

Exit les rapports sociaux ayant présidé à la formation des minorités - plurielles - canadiennes. Avec l'adoption de la politique sur le multiculturalisme, les minorités francophones deviennent des minorités comme les autres en compétition pour les ressources de l'État. L'adoption de la politique sur le multiculturalisme est donc un moment charnière pour les francophonies hors Québec qui sont ainsi l'objet d'un double mouvement simultané que Thériault et Meunier appellent "dénationalisation» et "ethnicisation ${ }^{6}:$ "On serait ainsi progressivement passé d'une logique nationale où le politique primait à une logique identitaire contenue et reproduite par un mode gestionnaire de la "différence culturelle et linguistique "» (Thériault et Meunier, 2008, p. 216). La Loi sur les langues officielles et les initiatives qui en découlent (article 23, programmes de soutien aux langues officielles, etc.) ne manquent pas de participer à ce double processus en dissociant la langue de la culture. Il n'y a plus deux langues et deux cultures nationales, mais deux langues officielles accueillant des cultures multiples. Même avec la récente Loi modifiant la Loi sur les langues officielles, les politiques linguistiques s'apparentent davantage à des mesures d'affirmation positive et de protection des langues officielles dans le cadre général du pluralisme culturel canadien qu'à des politiques statuant l'égalité juridique entre deux langues nationales.

Selon Juteau (1993, p.66), les rapports historiques conflictuels entre les deux peuples dits fondateurs du Canada ont fait «de 
l'ethnicité un réel critère d'identification, de revendication, d'action sociale et d'allocation de ressources matérielles et symboliques au Québec et dans l'ensemble du Canada ». À travers la loupe du multiculturalisme, les enjeux de société s'ethnicisent au détriment d'autres rapports d'inégalité sociale. C'est sans doute là un des premiers effets de la politique sur le multiculturalisme canadien sur les milieux intellectuels :l'émergence (d'abord dans les travaux des chercheurs états-uniens sur le Québec et l'Acadie) puis la prédominance au cours des années 1980 (dans les études menées par des Francophones canadiens) du paradigme ethnique pour penser l'identité des minorités francophones.

On ne sous-estimera pas la portée de la politique canadienne du multiculturalisme et de la Charte des droits dans le développement de la représentation et de la structuration identitaires des francophones minoritaires, voire de l'ensemble des groupements identitaires canadiens. [...] Hors de tout doute, la popularité du paradigme ethnique dans l'explication des processus identitaires des francophonies minoritaires doit beaucoup à cette nouvelle réalité. (Thériault et Meunier, 2008, p. 220)

Toutefois, contrairement à Thériault et Meunier, nous ne pensons pas que ce double processus de dénationalisation et d'ethnicisation des communautés francophones hors Québec institué par la conjugaison de la Loi sur les langues officielles et de la Politique sur le multiculturalisme a fait "place à des espaces linguistiques civiques, inclusifs $»^{7}$ (Thériault et Meunier, 2008, p. 229). Si ces caractères civiques et inclusifs sont bien contenus dans le principe, la Loi sur les langues officielles et la politique sur le multiculturalisme ont plutôt conduit les communautés francophones hors Québec à revêtir le dossard du minoritaire et à produire, du moins à leurs débuts, un discours homogénéisant, et donc excluant. Soucieuses, tout comme les minorités ethniques issues de l'immigration, de voir leur identité de minoritaire reconnue par l'État, elles sont elles aussi entrées dans la course aux subsides. Or, un tel jeu relationnel entre l'État et la population civile constituée, somme toute, en 
"La logique de régulation sociale instaurée par la Loi sur les langues officielles et la Politique sur le multiculturalisme, celle de communautés qui au mieux se côtoient poliment et au pire se font compétition pour leur reconnaissance identitaire, n'offre donc pas les conditions idéales pour une analyse de la simultanéité des processus pluriels de domination au sein d'une même catégorie de minoritaire. groupes d'intérêts mobilisés autour de l'affirmation d'identités, a pour effet d'encourager la production de discours identitaires excluant la différence, essentialisant, folklorisant et figeant ${ }^{8}$. La logique de régulation sociale instaurée par la Loi sur les langues officielles et la Politique sur le multiculturalisme, celle de communautés qui au mieux se côtoient poliment et au pire se font compétition pour leur reconnaissance identitaire, n'offre donc pas les conditions idéales pour une analyse de la simultanéité des processus pluriels de domination au sein d'une même catégorie de minoritaire. Le contexte invite moins à l'observation des inégalités sociales de toutes sortes (quelles soient basées sur le genre, la classe sociale, etc.) qu'à l'étude de l'histoire des groupes et à l'identification de leurs caractéristiques propres en vue de légitimer leur existence et leur aspiration à la reconnaissance :

Ces différents facteurs vont obliger les réflexions sur l'ethnie à certaines stratégies discursives pour rendre compte du fait identitaire francophone minoritaire. En effet, il a fallu : 1) d'abord que les discours sur l'ethnie se démarquent théoriquement de la tradition américaine; 2) ensuite, qu'ils se démarquent idéologiquement de la théorisation québécoise de l'identité nationale; 3 ) et qu'ils construisent, enfin, un ensemble discursif basé sur un "entre soi», créé à partir du lieu symbolique de la communauté elle-même (son histoire) et de ce qu'elle peut produire aux divers niveaux du socioculturel, $\mathrm{du}$ politique et de son propre regard sur elle-même. (Bernier, 1995, p. 53-54)

Aussi, selon Bernier (1995, p. 56), l'endossement du statut de minoritaire enferme-t-il ses protagonistes dans une logique d'homogénéisation et de lutte contre la fragmentation d'une identité collective jugée menacée.

Ce rapport dichotomique [dominant/dominé] que nous présentent les textes sur l'identité franco-ontarienne pourrait apparaitre réducteur 
si là ne résidait pas, précisément, selon eux, la seule expression possible de la réalité identitaire du minoritaire, l'expression d'un désespoir. Pris entre ces deux pôles, sur un même axe, comment le discours minoritaire peut-il, autrement qu'en les subsumant sous ses propres catégories, intégrer les réalités d'autres minoritaires??

Cela dit, si à ses premières heures le multiculturalisme canadien vise essentiellement à ne pas être assimilationniste et à faire du pluralisme une source de richesse nationale, il sera rapidement soumis à des critiques. Ses opposants lui reprochent en effet de folkloriser les cultures et d'ethniciser les individus en les réduisant à leur identité culturelle, sans pour autant corriger d'autres sources d'inégalités. C'est pourquoi, au cours des années 1980, l'État s'engagera sur la voie de la justice sociale :

À la fin des années 1980, la Loi sur le Multiculturalisme canadien entérine la politique de reconnaissance et d'aide aux langues, cultures et groupes minoritaires, mais soutient activement

"Avec l'adoption de la Loi sur le Multiculturalisme en 1988, les actions étatiques se déplacent donc progressivement vers des mesures plus générales de justice où d'autres groupes jugés défavorisés, notamment les Autochtones et les femmes, sont définis et ciblés selon la même approche que les groupes ethniques pour faire l'objet de mesures positives..." les mesures visant à contrer la discrimination, à promouvoir l'équité dans l'emploi, ou à accroître la participation politique et sociale. (Bertheleu, 2001, p. 37)

Avec l'adoption de la Loi sur le Multiculturalisme en 1988, les actions étatiques se déplacent donc progressivement vers des mesures plus générales de justice où d'autres groupes jugés défavorisés, notamment les Autochtones et les femmes, sont définis et ciblés selon la même approche que les groupes ethniques pour faire l'objet de mesures positives visant à réparer des injustices sociales au nom d'un principe d'équité ${ }^{10}$. Ainsi, d'une politique visant à ses débuts à reconnaitre les identités culturelles au nom de la diversité, nous passons avec la Loi sur le Multiculturalisme à une politique cherchant à reconnaitre le pluralisme au nom de la correction de la discrimination systémique dont sont l'objet certains groupes sociaux sur la base de leur appartenance culturelle. 
Si ce multiculturalisme canadien "réformé» cherche désormais à croiser les demandes de reconnaissance identitaire avec des revendications de justice et d'équité sociale, il semble toutefois y échouer en partie. Le multiculturalisme est souvent réduit, du moins dans les représentations sociales, à la question ethnique, voire à celle des "minorités visibles». En effet, dans leur rapport De la mosaïque à l'harmonie : le Canada multiculturel au XXI siècle publié en 2007 à l'issue de tables de consultation tenues dans huit villes canadiennes, Kunz et Sykes notent que la question de la diversité ne semble intéresser que les membres des minorités visibles puisque les Autochtones et les personnes ne faisant pas partie des dites minorités visibles ne se sont pas présentés aux discussions : "Cette situation renforce les perceptions voulant que le multiculturalisme s'adresse uniquement aux minorités visibles, ce qui a pour conséquence d'exacerber la dichotomie «nous et eux «» (Kunz et Sykes, 2007, p. 4). Des études réalisées en milieu scolaire au cours des décennies 1980 et 1990 montrent également que le vocabulaire employé dans le discours officiel d'ouverture à l'Autre est souvent, encore aujourd'hui, réducteur, culturalisant et misérabiliste (Bertheleu, 2001, p. 40).

Aussi, à travers la lunette des relations interethniques et des logiques de reconnaissance identitaire, la dimension ethnique des rapports de différenciation sociale tendrait encore aujourd'hui à faire écran aux autres sources d'inégalités et inciterait à réfléchir aux minorités les unes par rapport aux autres plutôt que comme pouvant s'emboîter les unes dans les autres. Malgré ces transformations en vue de croiser les demandes identitaires avec des demandes de justice sociale, il semble donc que le multiculturalisme canadien ait du mal à se départir de ses germes qui ethnicisent les enjeux sociaux et invisibilisent les autres rapports de différenciation sociale (de sexe, de classes, ou autres). ${ }^{11}$

Cela étant dit, rappelons que le multiculturalisme porte en lui ce que Fassin et Fassin (2006, p. 257) nomment le «paradoxe minoritaire": toute politique en faveur des minorités, plus particulièrement celle qui vise à la fois la reconnaissance identitaire et la justice sociale, a pour effet pervers de renforcer les catégorisations ethniques qu'elle a pour vocation de dépasser. 
«De fait, nous assistons depuis une dizaine d'années au Canada français, et ce, tant dans le champ scientifique que militant, à des tentatives de reformulation de l'identité francophone minoritaire dans une perspective plus inclusive des différences, notamment ethniques. "
Toutefois, nous rappellent Fassin et Fassin, les mesures d'affirmation positive permettent des transformations structurelles réelles qui ont des effets jusque dans les représentations que se fait la société ou une minorité — d'elle-même, auxquels effets les intellectuels n'échappent pas. En outre, le champ des études ethniques a lui-même changé, passant progressivement d'une approche essentialiste à une approche interactionnelle de la culture, basée notamment sur les frontières - changeantes - qui définissent les contours des groupes ethniques ${ }^{12}$. De fait, nous assistons depuis une dizaine d'années au Canada français, et ce, tant dans le champ scientifique que militant, à des tentatives de reformulation de l'identité francophone minoritaire dans une perspective plus inclusive des différences, notamment ethniques. L'identité de la francophonie minoritaire serait relationnelle, fluide et capable de métissage. Elle trouverait ses sources dans les foyers de socialisation multiples où elle s'actualise et que les réseaux sociaux et les flux culturels permettent de revigorer, freinant ainsi l'assimilation à la majorité anglophone (Farmer, Chambon et Labrie, 2003). Les défis sont grands, mais une "nouvelle francité" est possible (Labrie et Heller, 2003). Des travaux récents réalisés sur l'école et auprès de la jeune génération vont également dans ce sens (Gérin-Lajoie, 2006; Pilote, 2007). Un certain discours scientifique semble donc vouloir émerger et s'éloigner des discours identitaires initiaux homogénéisants - et potentiellement exclusifs.

Or, Thériault et Meunier condamnent ces analyses issues du paradigme ethnique, qu'ils disent proches des théories du choix rationnel et empreintes de l'idéologie pluraliste et d'une modernité radicale, car elles omettent de tenir compte de la spécificité nationalitaire des minorités francophones, si ce n'est qu'elles la dénigrent en l'associant à un traditionalisme désuet à l'heure de la mondialisation. Selon eux, de telles analyses menacent de fragmentation les francophonies minoritaires :

D'un point de vue individuel, l'engouement momentané pour une identité radicalement neuve à construire peut être perçu comme un gain; du point de vue collectif cependant, il est clair qu'un groupe nécessitant des relations 
affinitaires pour sa survie et son maintien est un groupe fragile, balloté au gré des modes et des tendances et traversé par des conflits. (Thériault et Meunier, 2008, p. 215)

Dès lors, devrait-on en conclure que les lois sur le multiculturalisme et sur les langues officielles nuisent aux francophonies en situation minoritaire? Si on suit le raisonnement de Thériault et Meunier, il semble en effet qu'en dépouillant l'identité francophone de son caractère national initial pour en faire un espace linguistique civique, pourvu désormais d'une mémoire ethnique, ces lois sonneraient le glas des communautés francophones en contexte minoritaire. Dans le cas où les intérêts de certaines catégories ou sous-groupes dominés entreraient en conflit avec les intérêts défendus dans le discours dominant des minorités francophones, cela conduit-il à dire que nous devrions établir et légitimer une sorte de hiérarchisation des minorités selon leur niveau d'historicité? ${ }^{13}$ Dans ce cas, que dire du niveau d'historicité des Autochtones? Et que faire des femmes ou des homosexuels, catégories de minoritaires également naturalisées par la discrimination? Ne pouvons-nous pas suggérer, inversement, que les gains obtenus par les communautés francophones en situation minoritaire, via notamment les politiques linguistiques, peuvent être des sources de domination pour d'autres minorités en leur sein?

Le débat n'est pas simple. Avant de tenter une réponse à ces questions délicates, voyons la deuxième raison de l'émergence tardive des travaux académiques s'attardant à la multiplicité et à la simultanéité des processus de minorisation au sein des minorités francophones.

\section{La pensée féministe au fondement des analyses «des minorités dans les minorités» francophones canadiennes}

Si les analyses des femmes en tant que minorité au sein des minorités francophones canadiennes voient le jour au cours 
«...ce sont les

féministes qui, de manière générale, investissent les premières l'étude de la pluralité des processus de domination en francophonies minoritaires en croisant les catégories minorisées "identité linguistique», "genre", "classe sociale" et "ethnicité»." des années 1980, et ce, parallèlement à leur plus grande visibilité politique ${ }^{14}$, celles s'attardant aux minorités ethniques — toujours au sein des communautés francophones minoritaires - apparaissent plus tardivement, soit vers la fin des années 1990 et le début des années $2000^{15}$. Toutefois, au-delà de cet exercice consistant à dater l'arrivée des recherches sur l'ethnicité en contexte francophone minoritaire, ce qui saute aux yeux du chercheur qui s'adonne à une revue de la littérature sur la question est que ce sont les féministes qui, de manière générale, investissent les premières l'étude de la pluralité des processus de domination en francophonies minoritaires en croisant les catégories minorisées «identité linguistique», "genre», «classe sociale» et «ethnicité» ${ }^{16}$.

Le constat n'est, au demeurant, pas si étonnant. Les théorisations pionnières de la féministe française Colette Guillaumin avaient ouvert la voie du couplage "femme» et «ethnicité », du moins parmi les rares ouvrages en français ${ }^{17}$, en montrant à travers sa théorisation du «sexage» que les deux questions dérivent d'un même mécanisme : «Ces catégories occupent une place spécifique dans les rapports sociaux, celle d'être considérées comme des catégories «naturelles «» (Guillaumin, 1992, p. 10). Sa théorie de l'oppression sera reprise à bon compte par Juteau-Lee et Roberts dans l'article "Ethnicity and Feminity : (d') après nos expériences» publié dans le numéro de Canadian Ethnic Studies / Études ethniques au Canada qu'elles coordonnent en 1981 et qui rassemblent des textes traitant de l'articulation entre les différents rapports de domination vécus par des femmes canadiennes (femmes des minorités francophones, des minorités "visibles", femmes autochtones, etc.). Selon Bernier (1995, p. 49), le double ancrage, tant de Guillaumin que de Juteau-Lee, dans le champ de connaissances des phénomènes ethniques et dans le mouvement féministe leur a permis d'avoir des regards scientifiques différents — de ceux prétendant à l'universalisme et à la neutralité — sur les mécanismes de production des identités et leur reconnaissance. Elles ont articulé deux champs de connaissances jusqu'alors abordés séparément, celui des études ethniques et celui des études des femmes, en montrant pour la première comment le servage des femmes dans l'économie domestique sert tant la domination 
"...les discours

intellectuels des

femmes en milieux

francophones

minoritaires mirent

du temps à sortir

de leur caractère

particulier pour trouver

une reconnaissance

au sein du milieu

académique...» masculine - le patriarcat - que le capitalisme et comment, pour la seconde, le champ des études ethniques a historiquement occulté la participation des femmes dans la production de l'ethnicité, à un point tel d'ailleurs que l'immigration et les phénomènes ethniques - discrimination, racisme, etc. — sont longtemps apparus comme des réalités strictement masculines.

Ainsi, c'est par le biais des travaux des féministes visant à sortir les femmes francophones de leur invisibilisation multiple - domination par les hommes francophones, subordination linguistique par la majorité anglophone, marginalisation sur la base d'une identité nationale canadienne-française déchue de la part des femmes québécoises - que les recherches en sciences sociales sur les rapports complexes et articulés de domination firent leur entrée en contexte francophone minoritaire canadien (Juteau-Lee et Roberts, 1981). La prise en compte de l'hétérogénéité ethnique de la catégorie «femme francophone vivant en milieu minoritaire" sera intégrée ici et là à partir du milieu des années 1990, particulièrement dans le champ du service social, mais force est de constater que les publications incluant les mécanismes de subordination sur une base ethnique au sein des communautés francophones minoritaires demeurent encore peu nombreuses ${ }^{18}$.

À partir de l'analyse d'un corpus de textes scientifiques faisant montre soit d'une analyse sociologique de l'identité francophone en Ontario, soit de l'identité des femmes francophones en situation minoritaire, Bernier (1995) montre bien comment les discours intellectuels des femmes en milieux francophones minoritaires mirent du temps à sortir de leur caractère particulier pour trouver une reconnaissance au sein du milieu académique, dominé par les productions d'auteurs masculins. Alors que le discours sur l'identité franco-ontarienne bénéficie déjà d'une reconnaissance dans le champ épistémologique de la scientificité pour que l'effet sociopolitique recherché soit visible, les discours scientifiques par et pour les femmes, avant d'être entendus dans le champ sociopolitique, ont d'abord dû conquérir le statut de productrices de savoir dans l'espace scientifique : 
[...] alors que le discours produit par les femmes francophones est largement tributaire du discours sur l'identité minoritaire, celui-ci en retour fait peu de cas de leurs productions. Il y a dépendance et non-échange; il y a, symboliquement, dans le champ du discursif sur la question des minoritaires francophones en Ontario un discours social accepté (celui de l'identité franco-ontarienne) qui se donne à voir, tant à lui-même qu'aux autres, comme représentation hégémonique et un discours «minoritaire» (celui des femmes francophones) qui est présenté comme particulier, spécifique, marginal, et donc la reconnaissance tarde à être établie. (Bernier, 1995, p. 57)

Bernier (1995) en tient pour preuve le peu de citations dont ont fait l'objet les analyses sociologiques de l'ethnicité de Juteau en Ontario français, et ce, jusqu'en 1990.

L'évolution des analyses féministes à la faveur d'une appréhension de l'imbrication des inégalités/identités multiples suit grosso modo les changements qui se sont opérés au sein du mouvement féministe pris globalement, notamment aux États-

"Avec la focalisation du regard sur les rapports sociaux de genre grâce aux luttes sociales et aux travaux des chercheuses féministes, il s'est avéré que d'autres modes de différenciation sociale passaient dans l'ombre..." Unis et en Grande-Bretagne. Avec la focalisation du regard sur les rapports sociaux de genre grâce aux luttes sociales et aux travaux des chercheuses féministes, il s'est avéré que d'autres modes de différenciation sociale passaient dans l'ombre, contrevenant ainsi à l'objectif prioritaire pourtant poursuivi visant à dévoiler tout un segment de la population - la moitié, dans ce cas-ci historiquement invisibilisé. De fait, il s'avérait que les femmes au nom desquelles on parlait et à qui on souhaitait rendre enfin et en toute légitimité leur rôle d'actrices de l'histoire étaient majoritairement des femmes blanches, occidentales, hétérosexuelles et de classe moyenne, et que les féministes noires s'en trouvaient par conséquent marginalisées ${ }^{19}$. Or, ces mêmes femmes noires se trouvaient également reléguées par les hommes noirs en périphérie du mouvement antiraciste afro-américain 
"...les analyses des différentes sources de domination qui avaient cours dans les années 1960 et 1970 présumaient qu'un mode de différenciation sociale avait la prédominance sur les autres, le plus souvent la classe sociale ou le genre. »
Black Power, cette fois non pas en vertu de leur race/ethnicité, mais en vertu de leur genre (Wallace, 1990). Donc, d'un côté comme de l'autre, au sein du mouvement féministe comme du mouvement antiraciste, ces femmes étaient invisibilisées puisque la conjonction de leurs identités/inégalités multiples n'était pas prise en compte.

Ces travaux des féministes noires états-uniennes et anglaises, parmi les premiers à rendre compte des inégalités multiples résultant de l'imbrication des rapports sociaux de classe, de genre et de race/ethnicité, ont donné naissance à un courant d'analyse qu'on appelle, dans les milieux académiques et militants anglosaxons, l'intersectionnalitée ${ }^{20}$. C'est Collins (2000, p. 252), l'une des penseuses du féminisme noir, qui parlera la première de l'intersectionnalité comme paradigme (Bilge, 2009, p. 71). Au Canada, l'expression "analyse intersectionnelle» est généralement associée à Stasiulis, qui l'emploie dans un article fort éclairant publié en 1999. Selon Bilge (2009, p. 71-72), c'est toutefois la politologue Hancock qui en propose une véritable théorisation en postulant qu'il faut dépasser l'intersectionnalité comme spécialisation ayant permis de mettre au jour le vécu des femmes qui sont situées à l'intersection de multiples rapports de domination, notamment des femmes noires, afin de l'appréhender comme un paradigme, c'est-à-dire comme «un ensemble de théorie normative et de recherche empirique» (Hancock, 2007, p. 250-251).

Qu'est-ce que l'intersectionnalité? Bilge $(2009$, p. 70$)$ en offre une définition succincte et claire :

L'intersectionnalité renvoie à une théorie interdisciplinaire visant à appréhender la complexité des identités et des inégalités par une approche intégrée. Elle réfute le cloisonnement et la hiérarchisation des grands axes de la différenciation sociale que sont les catégories de sexe/genre, classe, race, ethnicité, âge, handicap et orientation sexuelle.

De fait, les analyses des différentes sources de domination qui avaient cours dans les années 1960 et 1970 présumaient qu'un 
"...l'effet prééminent

d'un mode de

différenciation sociale

dépend des contextes

et de leurs effets

structurants, et ne

saurait en aucun cas

justifier le choix d'un

mode d'organisation

des sociétés comme

postulat à une théorie

générale de portée

universelle. » mode de différenciation sociale avait la prédominance sur les autres, le plus souvent la classe sociale ou le genre. En corollaire de ce postulat, on supposait que les sources de domination avaient des effets cumulatifs, les conséquences des sources «secondaires» venant s'ajouter aux effets de la principale forme d'inégalité sociale. C'est ainsi que certaines auteures parleront jusque dans les années 1980 de double ou triple statuts d'infériorité des femmes. Or, ces prémisses ont vite rencontré leurs limites qui, dans le cas des minorités francophones canadiennes, ont été soulevées dès le début des années 1990 (Cardinal, Lapointe et Thériault, 1990; Labelle, 1990). D'abord, l'effet prééminent d'un mode de différenciation sociale dépend des contextes et de leurs effets structurants, et ne saurait en aucun cas justifier le choix d'un mode d'organisation des sociétés comme postulat à une théorie générale de portée universelle. Si les femmes apparaissent reléguées, statistiquement, dans des emplois subalternes en Ontario francophone pris globalement, pour emprunter un exemple à Juteau-Lee et Roberts (1981, p. 9), il se peut qu'à Hearst, là où le français est plutôt assuré, mais où le marché du travail est encore majoritairement occupé par les hommes - rappelons que l'exemple est donné au début des années 1980 - ce soit effectivement en raison de leur identité de femmes; à Sudbury, en revanche, où l'anglais domine le marché du travail, il est possible que cela s'explique effectivement par leur identité linguistique.

Que les processus de minorisation soient multiples ne veut donc pas dire qu'ils se confondent ou se superposent. Il est impossible, en effet, d'affirmer que tous les francophones vivant en situation minoritaire se trouvent précarisés socialement et économiquement, bien qu'ils appartiennent à une catégorie sociale historiquement minorisée. Que dire, par exemple, des hommes et de la classe bourgeoise francophones? De même, il serait totalement injustifié de déclarer que tous les segments précarisés de la population canadienne se trouvent dans les milieux minoritaires francophones. Les classes ouvrières anglophones, les Autochtones, les femmes anglophones et québécoises, les minorités ethniques non francophones, pour ne donner que ces exemples, échappent alors tous à cette catégorie spécifique de 
population minorisée. La réalité sociale des individus, ainsi que les mécanismes sociaux qui la sous-tendent, sont pluriels et agissent selon plusieurs axes d'organisation sociale de manière simultanée et interactive.

La prise en compte simultanée des multiples systèmes d'oppression dans la (re)production des inégalités sociales d'une part, et le refus de leur hiérarchisation d'autre part - ou, pour être plus exact, du refus d'un classement hiérarchisé établi a priori et à vocation universelle ${ }^{21}$-, lesquels sont au fondement des théories intersectionnelles, appellent donc à la nécessité de contextualiser les phénomènes sociaux étudiés: «The contextualization involves recognizing that the meaning of a social location $^{22}$ - a social construction - is not static. It also involves recognizing that the salience of our various social locations, whether for ourselves or our social environment, is not static either" (Denis, 2008, p. 681).

Cette genèse des travaux féministes en francophonie minoritaire nous a permis de constater que c'est par leur intermédiaire que l'intersection des processus pluriels de minorisation a commencé de façon rigoureuse à être prise au sérieux dans les milieux scientifiques et militants. Nous avons vu qu'elle est étroitement liée à la genèse du féminisme en général, et à l'émergence du paradigme de l'intersectionnalité, lequel nous offre des pistes de réflexion quant à la manière d'appréhender les tensions pouvant exister entre les droits et intérêts des minorités francophones d'une part, et les droits et intérêts des autres minorités d'autre part. Pour ce faire, revenons à la politique sur le multiculturalisme et à la Loi sur les langues officielles, ainsi qu'au débat que nous avons soulevé à leur propos.

\section{Le multiculturalisme nuit-il aux minorités francophones (et vice versa)?}

Est-ce que la reconnaissance publique des minorités que favorise le multiculturalisme - avec les efforts de valorisation des 
identités et des différences auprès de l'État qui l'accompagnent, potentiellement exclusifs et essentialisants - ne risque pas de contrevenir aux luttes des francophones pour le maintien du fait français, si par conséquent leurs communautés sont sujettes à l'éclatement de leur projet politique et à la fragmentation des luttes sur des bases identitaires?

Inversement, on peut se demander si la défense des intérêts des minorités francophones ne nuit pas aux intérêts des autres minorités. Les pressions exercées par les communautés francophones minoritaires sur les classes défavorisées, sur les jeunes et sur les immigrants pour l'adoption du français — et plus exactement, d'un français jugé légitime par l'élite - ne risquent-elles pas d'entrer en tension avec les aspirations sociales et économiques des individus et familles concernés quand on sait, par exemple, que le bilinguisme est aujourd'hui un atout fort recherché dans les milieux canadiens du travail? De fait, des travaux ont documenté les transferts linguistiques des classes ouvrières francophones vers l'anglais en raison de cette domination de la part de l'élite au sein du réseau des écoles de langue française (Mougeon et Heller, 1986; Welch 1993). Selon Bélanger (2007, p. 53) : «L'école de langue française, du fait de sa situation en milieu minoritaire, est amenée à défendre une conception monolingue et homogénéisante, qui la rend de fait élitiste et la conduit à minorer les faits liés à la diversité». L'auteure poursuit en soulignant que sur le terrain, les enseignants attribuent souvent les difficultés d'apprentissage de la langue enseignée $e^{23}$ des élèves issus de l'immigration, y compris ceux en provenance d'Afrique francophone, à leur langue première (Bélanger, 2007, p. 56), comme s'il ne leur venait pas à l'idée que le français appris dans le pays d'origine puisse avoir sa légitimité. Dans un semblable ordre d'idées, Kérisit (1998) rappelle que le choix des nouveaux arrivants en Ontario français de suivre des cours d'anglais ou d'inscrire leurs enfants dans les écoles anglophones, plutôt que d'être un rejet de la cause francophone, peut tout simplement refléter une stratégie de survie sur un marché du travail encore largement fermé aux personnes unilingues françaises. Doit-on alors conclure que la poursuite des revendications et la défense des droits des minorités francophones 
canadiennes, grâce notamment à la Loi sur les langues officielles, contreviennent aux intérêts des autres minorités en leur sein, notamment ethniques? À force de vouloir protéger les droits et défendre les acquis linguistiques des minorités francophones, ne risque-t-on pas de négliger les intérêts des femmes, des jeunes, des classes ouvrières, des populations rurales, des gais et lesbiennes, des personnes avec un handicap, des immigrants et des communautés ethniques? L'enjeu n'est pas que militant et ne se réduit pas aux tensions soulevées entre divers types d'engagements sociaux et politiques. Il s'imprègne au plus profond des subjectivités, influe sur certains choix personnels, génère des conflits familiaux, cause des malaises identitaires.

En vérité, ces questions provocatrices, qui se veulent faire écho au titre d'Okin (1997) dans son article «Is Multiculturalism Bad for Women?» ${ }^{24}$, ont pour but de mettre en exergue le même «faux dilemme» (Delphy, 2006) qui a déjà été dénoncé en ce qui concerne la thèse de cette féministe américaine. Selon Okin (1997), la reconnaissance des droits des minorités culturelles nuit à la cause des femmes puisqu'elle sert les intérêts de ceux qui sont à la tête des organisations ethniques, c'est-à-dire les hommes. Selon elle, la reconnaissance des particularités culturelles renforce simultanément le patriarcat minoritaire et la vulnérabilité intragroupe, celle des femmes, des enfants et des homosexuels. Les adversaires des tribunaux pouvant arbitrer des affaires familiales en s'appuyant sur la charia - ces derniers ont failli voir le jour en Ontario au début des années 2000 - ont notamment eu recours à cette forme d'argumentation. Okin tranche la question en proposant qu'en cas d'opposition entre l'égalité des cultures entre société majoritaire et groupes ethniques minoritaires - et l'égalité des sexes, ce soit l'égalité des sexes qui devrait prévaloir. Dans un article intitulé «Is Feminism Bad for Multiculturalism?», Kukathas (2001) rétorque pour sa part en posant la question inverse. Il postule en effet qu'en poursuivant la défense des intérêts des femmes au nom d'un soi-disant universalisme, ce sont les intérêts des mouvements antiracistes, appuyés par les politiques sur le multiculturalisme, qui passent alors à la trappe.

Or, il s'avère que cette opposition féminisme/multiculturalisme 
fausse le débat en obligeant certains segments minoritaires des minorités ethniques - en l'occurrence, les femmes - à faire un choix impossible entre leur identité de femme et leur identité culturelle. Lorsqu'on continue d'opposer les droits dits universels des femmes aux droits dits particuliers des minorités culturelles et religieuses, c'est en effet la voix des femmes des minorités ethniques qui n'est pas entendue. Ces dernières se trouvent donc doublement opprimées : du fait de leur sexe et du fait de leur origine culturelle. En réalité, ces femmes font face à des «injonctions paradoxales» (Guénif Souilamas, 2003) : défendre les hommes de même appartenance ethnique contre le racisme dont ils sont victimes tout en souhaitant combattre le sexisme de ces derniers. C'est ainsi que des jeunes filles d'origine maghrébine en viennent à revendiquer leur virginité, non pas par soumission au père ou sous le poids d'une tradition culturelle oppressante, mais comme un moyen de ne pas renier les leurs (Hamel, 2006).

Si c'est une méprise d'opposer le féminisme au multiculturalisme, surtout lorsqu'on sait que les rapports de pouvoir constitutifs des majoritaires et des minoritaires sont situés et donc changeants, il serait tout autant erroné d'imposer un choix entre

"En considérant les intérêts des minorités francophones et les intérêts des autres minorités comme mutuellement exclusifs, ce sont les personnes appartenant à des minorités dans la minorité francophone qui disparaissent de l'analyse et des luttes sociales. " des minorités en concurrence. En considérant les intérêts des minorités francophones et les intérêts des autres minorités comme mutuellement exclusifs, ce sont les personnes appartenant à des minorités dans la minorité francophone qui disparaissent de l'analyse et des luttes sociales. Que dire par exemple de jeunes homosexuels de l'Ontario francophone qui se sentiraient opprimés à la fois au sein du mouvement gai de l'Ontario (anglophone) et du mouvement gai québécois, francophone? Pour paraphraser Bilge (2006b, p. 2), les mesures linguistiques visant à promouvoir la justice intergroupe - l'égalité entre les minorités francophones et la majorité anglophone - nécessitent d'être accompagnées par des mesures cherchant à favoriser la justice intragroupe, sur la base du genre, de l'orientation sexuelle, de la foi religieuse ou de l'appartenance ethnique. Ces deux visées doivent être menées conjointement si on veut éviter, d'une part, des actions linguistiques en faveur du développement de la francophonie, mais aveugles à l'exclusion d'autres groupes et, d'autre part, des 
«La contextualisation des multiples rapports de domination en présence permet de resituer au cour de l'analyse les rapports sociaux historiques ayant précédé la formation des francophonies minoritaires tout en mettant au jour les autres rapports de minorisation auxquels elles peuvent participer malgré elles." actions en faveur des minorités, particulièrement celles visées par le multiculturalisme, mais aveugles aux droits et aux revendications des communautés francophones hors Québec. La contextualisation des multiples rapports de domination en présence permet de resituer au cœur de l'analyse les rapports sociaux historiques ayant précédé la formation des francophonies minoritaires tout en mettant au jour les autres rapports de minorisation auxquels elles peuvent participer malgré elles.

Les recherches visant l'appréhension de l'imbrication des rapports sociaux de pouvoir, entre autres, grâce aux apports théoriques de l'intersectionnalité, peuvent nous aider à surmonter cette fausse alternative en donnant une voix à ces personnes qui se trouvent au confluent d'identités et d'inégalités multiples en contextes francophones minoritaires. L'une des visées de la recherche en sciences sociales, et c'est encore plus vrai en service social qui se donne pour mandat d'aider les individus, les groupes et les collectivités à répondre à leurs besoins et à surmonter leurs problèmes, devrait être de permettre aux personnes qui se trouvent à l'intersection de plusieurs identités minorisées, potentiellement en tension, d'éviter d'avoir à choisir entre une identité linguistique ou d'autres identités en les rendant visibles, en leur donnant une voix. Si le multiculturalisme canadien peut avoir comme effet pervers de renforcer la catégorisation des minoritaires et s'il a l'inconvénient d'encourager les minorités à une concurrence potentiellement stérile sur certains fronts de la lutte sociale, il offre, en revanche et paradoxalement, le cadre permettant l'entrée en scène des populations les plus marginalisées.

\section{Conclusion : de l'identitaire au minoritaire}

Est-ce que la reconnaissance sociale et identitaire d'un groupe situé au confluent d'une pluralité de rapports sociaux de différenciation - par exemple la reconnaissance de la communauté congolaise francophone de l'Ontario - ne risque pas de fragmenter à l'infini les minorités francophones? Que faire, en effet, des revendications potentielles des femmes congolaises francophones de l'Ontario? 
Et pourquoi ne pas pousser plus loin le raisonnement, et penser à l'éventualité d'un regroupement des femmes congolaises francophones lesbiennes de l'Ontario?

Probablement que les communautés francophones en situation minoritaire sont effectivement sujettes à la fragmentation, si on se résout à lire la réalité à travers une logique identitaire. Elles le sont sans doute moins, cependant, si on passe, comme le suggèrent Fassin et Fassin (2006, p. 251), d'une politique identitaire à ce qu'ils appellent une politique minoritaire :

En effet, qu'est-ce qu'une minorité? C'est une catégorie naturalisée par la discrimination. Si les communautés ont en partage une culture, ce qui définit les minorités, c'est l'assujettissement d'un rapport de pouvoir. Il ne s'agit certes pas de les opposer, mais de les distinguer : la minorité, à la différence de la communauté, n'implique pas nécessairement l'appartenance à un groupe et l'identité d'une culture; elle requiert en revanche l'expérience partagée de la discrimination. Les «Noirs» ou les «Arabes» en France, aujourd'hui, ont en commun non pas la "race», mais le racisme. C'est d'ailleurs également vrai, en parallèle avec les questions raciales, pour les minorités sexuelles - les femmes confrontées au sexisme ou les homosexuels à l'homophobie. Ce ne sont ni la nature, ni la culture qui sont au principe de la minorité, mais la naturalisation, fût-ce dans le registre culturaliste, d'une catégorie sociale par des pratiques discriminatoires.

Ce passage de l'identitaire au minoritaire n'est pas dépourvu d'intérêt politique pour les minoritaires francophones. Avec une politique de stricte reconnaissance des identités — sans égard aux injustices sociales ${ }^{25}-$, chaque groupe lutte effectivement pour le maintien et le développement de sa propre identité, parfois ouvertement les uns contre les autres. Avec une politique minoritaire, que l'on soit minorité nationale ou minorité 
"Non seulement une politique minoritaire s'ouvre-t-elle aux minorités dans leur diversité, mais aussi à tous ceux qui, peu importe d'ailleurs qu'ils fassent partie du groupe majoritaire, voudraient combattre les discriminations."

"C'est faire fausse route que de hiérarchiser les identités collectives, $y$ compris selon leur niveau d'historicité, parce que les réalités sociales observées sont d'une complexité trop grande et mouvante pour s'y laisser réduire." ethnique d'ailleurs, on s'engage "dans une critique transversale des assignations normatives» (Fassin et Fassin, 2006, p. 251). Non seulement une politique minoritaire s'ouvre-t-elle aux minorités dans leur diversité, mais aussi à tous ceux qui, peu importe d'ailleurs qu'ils fassent partie du groupe majoritaire, voudraient combattre les discriminations. Aussi, si on peut déplorer avec Thériault et Meunier (2008) que la logique qui anime actuellement les communautés francophones minoritaires soit davantage néocorporatiste, organisationnelle et lobbyiste que politique ${ }^{26}$, et s'il est légitime de prêcher comme eux pour le retour d'un projet politique chez les francophonies minoritaires, il est plus difficile de les suivre quant au ressort qui, selon eux, pourrait permettre cette renaissance d'une communauté politique : moins qu'une mémoire nationalitaire, somme toute relativement exclusive de ceux qui ont d'autres mémoires, c'est davantage le partage d'une discrimination basée sur la langue, voire l'expérience partagée bien que dans des contextes différents - du mépris de son identité collective, de sa culture de minoritaire, qui sont susceptibles de fonder un "nous", un désir de "faire société", une société plus juste et équitable. Dans cette perspective qui permet de penser la transversalité, des alliances entre organisations ayant des intérêts communs, suivant les moments et les lieux de la lutte sociale, sont admises. À travers le prisme d'une politique minoritaire - de la question sociale - des individus aux identités différenciées peuvent trouver le socle commun afin de s'unir, le temps d'une lutte, et formuler des revendications sur le plan politique.

Le minoritaire ne doit pas être postulé a priori, comme s'il s'agissait d'un état ou d'un statut. Il ressortit plutôt à une relation qui est transformable historiquement, voire changeante selon les contextes et les termes de la relation - le pouvoir, nous dit Foucault (1976), n'est pas un attribut, il est situationnel et relationnel. C'est faire fausse route que de hiérarchiser les identités collectives, y compris selon leur niveau d'historicité, parce que les réalités sociales observées sont d'une complexité trop grande et mouvante pour s'y laisser réduire. Il n'y a pas lieu de choisir entre deux identités - voire plus - en tension parce que ceux qui sont pris dans cette tension récusent eux-mêmes la 
plupart du temps cette obligation de choix ${ }^{27}$. L'idée n'est pas de refuser tout universalisme pour privilégier le relativisme, mais de ne prendre ni le parti de l'un, ni le parti de l'autre. Au contraire, en contextualisant les phénomènes sociaux étudiés, on se donne les moyens de contrer les forces idéologiques, qu'elles soient universalistes ou pluralistes, en dévoilant la multiplicité des rapports sociaux de domination en jeu à un moment et en un lieu donnés. Une telle lecture des réalités sociales s'accorde d'ailleurs avec une reconnaissance du caractère multiforme de «la» francophonie minoritaire canadienne ${ }^{28}$.

Plaider pour une pensée de la complexité (Fassin et Fassin, 2006), c'est cependant choisir la complexification des approches: tout le défi scientifique, tant théorique que méthodologique, réside en effet dans la capacité d'articuler les différents rapports sociaux de différenciation sociale et d'en offrir une interprétation. Le paradigme de l'intersectionnalité, pour reprendre cet exemple, soulève d'ailleurs actuellement nombre de débats et controverses, même parmi ceux qui s'en réclament (Bilge, 2009). Chose certaine, si on souhaite se positionner contre un modèle analytique qui hiérarchise a priori les appartenances pour privilégier la contextualisation des rapports sociaux de domination, il faut mettre en place des dispositifs méthodologiques qui permettent de connaitre au plus près à la fois ceux dont on parle souvent dans les journaux, mais dont on n'entend pas la voix, et les mécanismes sociaux plus larges qui engendrent ces catégorisations. Les intervenants et les chercheurs en service social sont parmi ceux qui ont cette proximité avec ces personnes et qui peuvent par leur travail leur donner la parole.

\section{Notes}

Je remercie Caroline Bouchard, étudiante à la maîtrise à l'École de service social de l'Université d'Ottawa, de son aide plus qu'appréciable dans la recherche documentaire. Je tiens également à remercier les deux personnes qui ont évalué ce texte pour leurs judicieux commentaires. Ils m'ont permis de lui apporter quelques précisions.

2 Une pléthore de littérature traite de la question identitaire chez les francophonies minoritaires canadiennes. À titre indicatif seulement, on pourra se référer aux ouvrages suivants : Louder et Waddel (1983); Daigle (1993); Jaenen (1993); Boudreau et Nielson (1994); Cotnam, Frenette et 
Whitfield (1995); Juteau (1999); Heller et Labrie (2003); Thériault (2007); Thériault, Gilbert et Cardinal (2008).

3 À l'exception des écrits sur le genre et la classe sociale, qui apparaissent dans les années 1980, les recherches s'intéressant à l'ethnicité se multiplient principalement dans la seconde moitié de la décennie 1990.

4 Lequel, d'ailleurs, est moins le fait d'une construction volontaire que d'un mouvement historique désordonné, fait de l'entrelacement des conjonctures économiques et démographiques d'une part, et des conflits d'acteurs sociaux bien réels, d'autre part (Bayart, 1996, p. 12).

5 Pour une genèse du rôle de l'Église comme principe spirituel instituant la «nation " canadiennefrançaise, voir Thériault et Meunier (2008), plus particulièrement les pages 224 à 228.

6 L'assignation à un statut de minoritaire au milieu d'autres minoritaires dépouille les francophonies minoritaires de leur caractère nationalitaire et renvoie leur identité mémorielle, leur particularisme, à de l'ethnique. En passant de nationale à minoritaire, leur référence identitaire subit une «désubstantialisation» et une "dépolitisation» (Thériault et Meunier, 2008, p. 218).

7 Et nous ne condamnons pas, comme le font ces auteurs, le passage d'un modèle d'identité nationaliste à une identité se voulant civique, fût-elle ethnique, qu'auraient opéré les adoptions consécutives de la loi sur les langues officielles et la politique sur le multiculturalisme.

8 C'est là l'un des reproches maintes fois adressés au multiculturalisme (Bertheleu, 2001;Wieviorka, 2005).

9 On peut émettre l'hypothèse que le travail sémantique de définition de soi des francophonies hors Québec comme minorités fut d'autant plus homogénéisant et exclusif, au début, qu'il s'accompagnait du traumatisme de la perte du statut privilégié de communauté d'histoire, de nation cofondatrice du Canada.

10 Le principe d'équité est entendu, dans ce cadre, comme le fait de donner des moyens préférentiels à des groupes défavorisés afin qu'ils puissent bénéficier de la même égalité des chances que les autres. Pour plus détails sur les liens entre équité et égalité, souvent à la source des débats opposant les tenants du multiculturalisme et ceux d'un modèle républicain d'intégration, voir Wieviorka (2005, p. 92).

11 Soulignons cependant que le multiculturalisme ne possède pas l'apanage de l'ethnicisation des enjeux de société. En France, où le modèle républicain d'intégration est souvent présenté comme étant aux antipodes d'un modèle multiculturaliste dit anglo-américain, l'existence et le recours ordinaire à des référentiels raciaux sont également observables et sont, ce qui est nouveau, de plus en plus reconnus par les dirigeants politiques et les milieux intellectuels confondus (Fassin et Fassin, 2006).

12 Pour plus d'informations sur l'évolution des théories de l'ethnicité, voir Poutignat et StreiffFeinart (1995) et Juteau (1999).

13 Thériault et Meunier soutiennent que le statut de minoritaire des francophones hors Québec les plonge dans la mer majoritaire avec d'autres minorités et conduit à l'effacement de la communauté comme communauté politique. Par conséquent, «la mémoire entretenue semble peu avoir de fonction proprement politique, car elle s'affirme dans une société qui ne saurait reconnaitre "aucune préminence morale» [souligné par nous] aux communautés d'histoire - même celles qui rappellent le courage des premières nations ou la détermination des Canadiens français 
(Beauchemin, 2004a, p. 169)» (Thériault et Meunier, 2008, p. 206). La légitime hiérarchisation a priori des groupes est également sous-entendue lorsqu'ils affirment que la majorité des minoritaires ne saurait équivaloir à la majorité des majoritaires (Thériault et Meunier, 2008, p. 217).

Cardinal (1995) établit la naissance d'un mouvement féministe franco-ontarien au milieu des années 1970.

15 À l'exception du texte fondateur de Juteau-Lee et Roberts (1981), sur lequel nous reviendrons, et de celui de Coulombe (1985).

16 Le texte de Dennie (1978), qui montre comment la société franco-ontarienne n'est pas homogène, mais traversée par des relations de pouvoir fondées sur la classe sociale, en constitue, à notre connaissance, une exception.

17 L'historienne et anthropologue américaine Ann Laura Stoler avait déjà relu les questions sexuelles de Foucault à l'aune des questions raciales pour en montrer leur inextricable enchevêtrement (Stoler, 1995).

18 Pour une revue de la littérature plus complète des féministes canadiennes ayant traité de l'imbrication des rapports de domination fondés sur le genre et l'ethnicité en contexte francophone minoritaire, se référer à Denis (2008). Depuis le milieu des années 1990, des travaux se sont intéressés à l'hétérogénéité ethnique des communautés francophones hors Québec, dans une perspective féministe et non féministe, parfois même en y incluant une troisième source de discrimination; on trouve notamment le numéro de la revue Reflets dirigé par Kérisit, Mianda et Dubois (1998) consacré à l'intervention en contextes minoritaires ainsi que d'autres publications scientifiques qui se trouvent dans notre bibliographie, précédées d'un astérisque.

19 Cet état de fait a été bien documenté par bell hooks (1992), notamment.

20 Bilge (2009, p. 74) souligne cependant que si «la parenté entre l'intersectionnalité et la pensée féministe noire semble largement acceptée", on a critiqué la trop forte association qui était parfois faite entre les deux en arguant l'évacuation de la contribution des féministes socialistes et marxistes européennes des années 70, lesquelles avaient observé l'articulation des rapports de domination genre/classe sociale. Au Canada, nous savons, avec Denis (2008), que les travaux de certaines féministes canadiennes s'inscrivaient dans une perspective intersectionnelle avant le nom.

21 Ce n'est pas la même chose de refuser toute hiérarchisation en tout temps ou d'admettre une hiérarchie selon les contextes. La non-hiérarchisation "absolue ", c'est-à-dire décidée a priori et non après vérification empirique, est remise en cause par certains auteurs qui craignent justement une décontextualisation et une anhistoricisation des phénomènes sociaux observés. Pour plus d'informations à ce sujet, voir Bilge (2009, p. 79).

22 L'auteure privilégie ce concept à celui de statut social, moins dynamique.

23 C'est nous qui soulignons.

24 La question n'est pas illégitime quand on sait, comme le montre Bilge (2006a) à l'aide de jugements de cour ayant effectivement eu lieu au Canada, que la volonté contenue dans le multiculturalisme d'accommoder les pratiques judiciaires à la différence ethnique conduit certains juges à atténuer les peines encourues par des hommes de minorités ethniques ayant commis un viol sur une femme de même appartenance ethnique au nom de leur culture d'origine. Cette "cultural defence», pourtant non formalisée dans le Common law, donne cours à la circulation et 
au renforcement des stéréotypes culturels les plus fầcheux (ex. : fierté virile des hommes, hyper sensualité des jeunes femmes, etc.) et participe à l'homogénéisation et à l'essentialisation des cultures des minorités ethniques.

25 Contre les critiques qui pourraient nous être adressées d'appréhender les francophonies minoritaires à partir du modèle ethnique plutôt que nationalitaire, nous précisons que nous nous situons sur le terrain de la question sociale. Nous ne distinguons pas les demandes identitaires des demandes sociales. Aussi, il n'est peut-être pas inutile de rappeler ici que le multiculturalisme canadien, depuis l'adoption de la Loi en 1988, et malgré qu'il y ait sans doute place à l'amélioration de ses applications, ne se réduit pas à la simple reconnaissance des identités. La Loi sur le multiculturalisme accorde une reconnaissance aux identités dans le but de combattre les inégalités sociales.

26 Cette tendance n'est pas qu'impulsée par le multiculturalisme, mais ressortit aussi, comme le signalent eux-mêmes les auteurs, «à une mouvance profonde de nos sociétés qui tend à définir le politique d'une manière essentiellement procédurale et juridique» (Thériault et Meunier, 2008, p. 229).

27 Les jeunes filles françaises d'origine maghrébine rencontrées par Guénif Souilamas (2003) et Hamel (2006), auxquelles nous avons fait référence précédemment, témoignent, par leurs stratégies, de ce renoncement à choisir. Le refus de devoir s'identifier en choisissant ou bien leur identité canadienne, ou bien leur identité culturelle d'origine est également bien présent dans de nombreuses recherches menées auprès de jeunes canadiens issus de l'immigration. Pour une revue de cette littérature, voir Karpinski (2008).

28 Reconnaissance à laquelle appelle Juteau (1994, p. 43) pour une meilleure compréhension «des» francophonies minoritaires : «Nous continuerons à tracer le lien entre les formes multiples des francophonies minoritaires et les multiples formes de leurs pouvoirs [...]. Nous découvrirons ainsi la diversité de leurs chants, de leurs paroles, de leurs projets, de leurs revendications. Nous contesterons les pratiques et les idéologies qui cherchent à hiérarchiser les divers types de communautés d'histoire et de culture et à exclure et mépriser les sans-État».

\section{Bibliographie}

^AMAL, Madibbo (2005), Immigration, race and language : black francophones of Ontario and the challenges of integration, racism and language discrimination, Ceris working paper $\mathrm{N}^{\circ} 38$, Ryerson University.

ANDERSON, Benedict (1996). L'imaginaire national : Réflexions sur l'origine et l'essor du nationalisme, Paris, La Découverte, 215 p.

BAYART, Jean-François (1996). L'illusion identitaire, Paris, Fayard, 306 p.

BÉLANGER, Nathalie (2007). «Une école, des langues...? L'enseignement du français en milieu minoritaire en Ontario ", Le français aujourd'hui, Vol. 3, No 158, p. 49-57.

^BELKHODJA, Chedly (2008). «Immigration et diversité au sein des communautés francophones en situation minoritaire", Thèmes canadiens, Université de Moncton, Centre Métropolis Atlantique, $136 \mathrm{p}$.

^BER GER, Marie Josée (automne 1997). «À la recherche d'une intégration», Reflets, Revue ontaroise d'intervention sociale et communautaire, Vol. 3, $\mathrm{N}^{\circ}$ 2, p. 74-86. 
BERNIER, Christiane (1995). «Femmes et ethnicité en Ontario français : limites des convergences théoriques des discours de l'identité", Recherches sociographiques, Vol. 8, No 2, p. 47-66.

BERTHELEU, Hélène (2001). «La politique canadienne du multiculturalisme : citoyenneté, accomodements institutionnels et équité», Sociétés contemporaines, $N^{\circ} 43$, p. 31-51.

BILGE, Sirma (2009). «Théorisations féministes de l'intersectionnalité », Diogène. Revue internationale des sciences humaines, $\mathrm{N}^{\circ} 225$, janvier-mars 2009, p. 70-88.

BOUCHARD, Louise, et collab. (2006). "Capital social, santé et minorités francophones», Revue canadienne de santé publique, volume 97, supplément 2, mai-juin 2006, p. 17-21.

BOUDREAU, Françoise (automne 1999). Langue minoritaire et services de santé mentale en l'an 2000 : droits et besoins des francophones de Toronto. Reflets, revue ontaroise d'intervention sociale et communautaire, Vol. 5, No 2, p. 123-154.

BOUDREAULT, Françoise, et Greg Marc NIELSON (dir.) (1994). «Les francophonies nord-américaines», Sociologie et Sociétés, Vol. 26, № 1, Montréal, Presses de 1'Université de Montréal.

CARDINAL, Linda (1995). «Making a difference :The theory and practice of francophone women's groups 1969-1982», dans Joy PARR (dir.), A diversity of women : Ontario 1945-1980, Toronto, University of Toronto Press, p. 281-316.

CARDINAL, Linda, Jean LAPOINTE et Joseph Yvon THÉRIAULT (1990). Individu, société et politique. La sensibilité des années quatre-vingt au sein de la recherche relative aux communautés francophones hors Québec, Document préparé pour la Fédération des jeunes canadiens-français pour le projet Vision d'avenir, $172 \mathrm{p}$.

CODERRE, Cécile, et Madeleine DUBOIS (automne 2000). «Solidarité et citoyenneté : initiatives pour contrer la pauvreté chez les francophones dans Ottawa-Carleton", Reflets, revue ontaroise de l'intervention sociale et communautaire, Vol. 6, $\mathrm{N}^{\circ}$ 2, p. 61-86.

COLlinS, Patricia Hill (2000). Black Feminist Thought : knowledge, Consciousness, and the Politics of Empowerment, New York, Routledge, 335 p.

COTNAM, Jacques, Yves FRENETTE et Agnès WHITFIELD (dir.) (1995). La francophonie ontarienne : bilan et perspectives de recherches, Hearst, Le Nordir., $361 \mathrm{p}$.

COULOMBE, Danielle (1985). «Doublement ou triplement minoritaire», Revue de l'Université d'Ottawa, Vol. 55, № 2, p. 131-136.

DAIGLE, Jean (dir.) (1993). L'Acadie des Maritimes :études thématiques des débuts à nos jours, Moncton, Chaire d'études acadiennes, $908 \mathrm{p}$.

DELPHY, Christine (2006). «Antisexisme ou antiracisme? Un faux dilemme», Nouvelles Questions féministes, Vol. 25, No 1, p. 59-83.

DENAULT,Anne-Andrée, et Linda CARDINAL (1999). «L'équité en matière d'emploi en Ontario et les francophones ", Recherches sociographiques, Vol. 40, No 1, p. 83-101.

DENIS,Ann (2008). «Intersectional Analysis :A Contribution of Feminism to Sociology», International Sociology, Vol. 23, No 5, p. 677-694.

DENNIE, Donald (1978). "De la difficulté d'être idéologue franco-ontarien», Revue du Nouvel Ontario, $\mathrm{N}^{\circ}$ 1, p. 69-90.

FARMER, Diane,Adrienne CHAMBON et Normand LABRIE (2003). «Urbanité et immigration : étude de la dynamique communautaire franco-torontoise et des rapports d'inclusion et d'exclusion", Francophonies d'Amérique, 16, p. 97-106. 
FASSIN, Didier, et Éric FASSIN (2006). «Conclusion. Éloge de la complexite », dans Didier FASSIN et Éric FASSIN (dir.), De la question sociale à la question raciale? Représenter la société française, Paris, La Découverte, p. 249-259.

FOUCAULT, Michel (1976). La volonté de savoir, Paris, Gallimard, 211 p.

*GÉRIN-LAJOIE, Diane (1995). «Les écoles minoritaires de langue française canadiennes à l'heure du pluralisme ethnoculturel", Études ethniques au Canada/Canadian Ethnic Studies, Vol. 27, № 1, p. 32-47.

*GÉRIN-LAJOIE, Diane (2002). "Le personnel enseignant dans les écoles minoritaires de langue française", dans Donatille MUJAWAMARIYA (dir.), L'intégration des minorités visibles et ethnoculturelles dans la profession enseignante : récits d'expérience, enjeux et perspectives, Montréal, Les Éditions Logiques, p.167-181.

*GÉRIN-LAJOIE, Diane (2006). «La contribution de l'école au processus de construction identitaire des élèves dans une société pluraliste", Éducation et francophonie,Vol. 34, printemps, p. 1-7.

*GÉRIN-LAJOIE, Diane, et Marianne JACQUET (2008). «Regards croisés sur l'inclusion des minorités en contexte scolaire francophone minoritaire au Canada", Éducation et francophonie, Vol. 36, No 1, p. 25-43.

GUÉNIF SOUILAMAS, Nacira (2003). Des beurettes, Paris, Hachette Littérature, 362 p.

GUILLAUMIN, Colette (1972). Idéologie raciste, genèse et langage actuel, Paris/La Haye, Mouton, 247 p.

GUILLAUMIN, Colette (1992). Sexe, race et pratique du pouvoir. L'idée de Nature, Paris, Côté-femmes, $239 \mathrm{p}$.

HAMEL, Christelle (2006). «La sexualité entre sexisme et racisme : les descendantes de migrant-e-s du Maghreb et la virginité», Nouvelles Questions féministes, Vol. 25, No 1, p. 41-58.

HANCOCK, Ange-Marie (2007). "Intersectionnality as a Normative and Empirical Paradigm», Politics \& Gender,Vol. 3, No 2, p. 248-254.

KARPINSKI,Julia (2008). Le discours scientifique au Canada sur l'identité ethnique et l'intégration des jeunes de la deuxième génération immigrante. Une analyse historique des représentations et des enjeux de pouvoir du savoir scientifique, Mémoire de maîtrise, École de service social, Université d'Ottawa, 94 p.

hooks, bell (1992). Black looks : race and representation, Toronto, Between the Lines, $200 \mathrm{p}$.

JAENEN, Cornelius J. (dir.) (1993). Les Franco-Ontariens, Ottawa, Presses de l'Université d'Ottawa, $443 \mathrm{p}$.

JUTEAU, Danielle (1993). «Multiculturalisme, interculturalisme et production de la nation», dans Martine FOURIER et Geneviéve VERMÈS (dir.), Ethnicisation des rapports sociaux. Racismes, nationalismes, ethnicismes et culturalismes, Éditions Fontenay/Saint-Cloud, Éditions L'Harmattan, Paris, p. 55-72.

JUTEAU, Danielle (1994). "Essai - Multiples francophonies minoritaires : multiples citoyenneté", Sociologie et Sociétés,Vol. 26, No 1, p. 33-45.

JUTEAU, Danielle (1999). L'ethnicité et ses frontières, Montréal, Presses de 1'Université de Montréal, $226 \mathrm{p}$.

JUTEAU-LEE, Danielle (1981). «Visions partielles, vision partiales : visions (des) minoritaires en sociologie», Sociologie et Sociétés, Vol. 13, No 2, p. 33-47.

JUTEAU-LEE, Danielle, et Barbara ROBERTS (1981). «Ethnicity and Feminity : (d') après nos expériences", Canadian Ethnic Studies/Études ethniques au Canada,Vol. 13, No 1, p. 1-23. 
JUTEAU-LEE, Danielle, et Jacques LAPOINTE (1983). «From French Canadians to FrancoOntarians and Ontarois : New Boundaries, New Identities", dans Jean Leonard Elliott (dir.), Two Nations, Many Cultures : Ethnic Groups in Canada, Scarborough, Prentice-Hall, $2^{\mathrm{e}}$ éd., 395 p.

*KÉRISIT, Michèle (printemps 1998), "Les défis de l'intervention interculturelle en milieu minoritaire de langue française en Ontario", Reflets, Revue ontaroise d'intervention sociale et communautaire, Vol. 4, No 1, p. 75-99.

^KÉRISIT, Michèle, Gertrude MIANDA et Madeleine DUBOIS (printemps 1998). «Intervention en contextes minoritaires", Reflets, Revue ontaroise d'intervention sociale et communautaire, Vol. 4, $\mathrm{N}^{\circ} 1$, printemps.

KUKATHAS, Chandran (2001). «Is Feminis bad for Multiculturalism», Public Affairs Quarterly, Vol. $15, N^{\circ} 2$, p. 83-98.

KUNZ, Jean Lock, et Stuart SYKES (2007). De la mosaïque à l'harmonie : le Canada multiculturel au XXIe siècle. Résultat des tables rondes, Projet de recherche sur les politiques, Ottawa, Gouvernement du Canada, $30 \mathrm{p}$.

LABELLE, Micheline (1990). "Femmes et migration au Canada : bilan et perspectives", Canadian Ethnic Studies, Vol. 22, № 1, p. 67-82.

LABRIE, Normand, et Monica HELLER (2003). «La francité ré-imaginée», dans Monica HELLER et Normand LABRIE (dir.), Discours et identités. La francité canadienne entre modernité et mondialisation, Cortil-Wodon, Éditions modulaires européennes, p. 403-419.

*LAFONTANT,Jean (1992). "Adieu ethnicité, bonjour minorités», Cahiers franco-canadiens de l'ouest, Vol. 4, No 2, p. 219-242.

*LAMINE Diallo, et Ginette LAFRENIÈRE (printemps 1998), «Stratégies pour la redéfinition de l'espace francophone à Sudbury", Reflets, Revue ontaroise d'intervention sociale et communautaire, Vol. 4 No 1, p. 140-150.

*LEGAULT, Marie-Josée (printemps 2005). «Les francophones d'Ottawa : une communauté diverse », Reflets, Revue ontaroise d'intervention sociale et communautaire,Vol. 11, № 1, p. 165-177.

LOUDER, Dean, et Eric WADDELL (dir.) (1983). Du continent perdu à l'archipel retrouvé : le Québec et l'Amérique française, Québec, Presses de l'Université Laval, 292 p.

MOUGEON, Raymond, et Monica HELLER (1986). «The social and historical context of minority French language education in Ontario", Journal of Multilingual and Multicultural Development, Vol.7, No 2 et 3, p. 199-227.

*MUJAWAMARIYA, Donatille (automne 2000). «Ils ne peuvent pas enseigner dans nos écoles : le dilemme des étudiants-maitres des minorités visibles nés au Canada", Reflets, Revue ontaroise d'intervention sociale et communautaire, Vol. 6, $\mathrm{N}^{\circ}$ 2, p. 138-165.

^NDUWIMANA, Mathilde, et Alice HOME (printemps 1995). «Vers des services attirants, accessibles et pertinents pour les personnes âgées immigrantes francophones?», Reflets, Revue ontaroise d'intervention sociale et communautaire, Vol. 1, No 1, p. 92-121.

OKIN, Susan Moller (1997). «Is Multiculturalism Bad for Women?», Boston Review,Vol. 22, Oct./ Nov. 1997.

PILOTE, Annie (2007), «Construire son identité ou reproduire la communauté? Les jeunes et leur rapport à l'identité collective», dans Michel BOCK (dir.), La jeunesse au Canada français : formation, mouvements et identité, Ottawa, Presses de l'Université d'Ottawa, p. 83-112. 
^PIQUEMAL, Nathalie, et Bathélémy BOLIVAR (2009). «Discontinuités culturelles et linguistiques : Portraits d'immigrants francophones en milieu minoritaire ", Journal of Internationa Migration and Integration, 10, p. 245-264.

POUTIGNAT Philippe, et Jocelyne STREIFF-FEINART (1995). Théories de l'ethnicité, Paris, Presses Universitaires de France, $270 \mathrm{p}$.

$\mathrm{RAE}, \mathrm{Bob}$ (2005). L'Ontario : chef de file en éducation. Rapport et recommandations, Gouvernement de l'Ontario, Imprimeur de la Reine pour l'Ontario, février, 129 p.

STASIULIS, Daiva (1999). «Feminist Intersectional Theorizing», dans Peter LI (dir.), Race and Ethnic Relations in Canada, Toronto, Oxford University Press, p. 347-397.

STOLER, Ann Laura (1995). Race and Education of Desire. Foucault's History of Sexuality and the Colonial Order of Things, Durhamand London, Duke U.P., 237 p.

THÉRIAULT, Joseph Yvon (1994). «Entre la nation et l'ethnie : sociologie, société et communautés minoritaires francophones", Sociologie et Sociétés, Vol. 26, No 1, p. 15-32.

THÉRIAULT, Joseph Yvon (2007). Faire société. Société civile et espaces francophones, Sudbury, Prise de parole, $384 \mathrm{p}$.

THÉRIAULT, Joseph Yvon, et E.-Martin MEUNIER (2008). «Que reste-t-il de l'intention vitale du Canada français?», dans Joseph Yvon THÉRIAULT, Anne GILBERT, et Linda CARDINAL (dir.), L'espace francophone en milieu minoritaire au Canada. Nouveaux enjeux, nouvelles mobilisations, Montréal, Fides, p. 205-238.

THÉRIAULT, JosephYvon,Anne GILBERT et Linda CARDINAL (dir.) (2008), L'espace francophone en milieu minoritaire au Canada. Nouveaux enjeux, nouvelles mobilisations, Montréal, Fides, 562 p.

WALLACE, Michelle (1990). Black Macho and the Myth of the Superwoman, London, New-York, Verso, $228 \mathrm{p}$.

WELCH, David (1993). "La lutte pour les écoles secondaires franco-ontariennes : une nouvelle perspective", Revue du Nouvel-Ontario, № 13 et 14, p. 109-131.

WIEVIORKA, Michel (2005). La différence. Identités culturelles : enjeux, débats et politiques, Paris, Éditions de l'aube, $201 \mathrm{p}$. 